\title{
The Occlusal Aspect of Children with Posterior Cross Bite in Deciduous Dentition Stage
}

\author{
Ryuichiro Takazawa, ${ }^{1}$ and Akira Usui ${ }^{2}$
}

${ }^{1}$ Nihon University Graduate School of Dentistry at Matsudo, Pediatric dentistry, Matsudo, Chiba 271-8587, Japan

${ }^{2}$ Department of Pediatric dentistry, Nihon University School of Dentistry at Matsudo, Matsudo, Chiba 271-8587, Japan

Correspondence to :

Ryuichiro Takazawa

E-mail : ponausu@gmail.com

Keywords :

Posterior cross bite, deciduous dentition, molar tooth axis, dental model, postero-anterior cephalogram

\begin{abstract}
Posterior cross bite in the deciduous dentition stage is unlikely to heal on its own even as the jaw grows. If left untreated, it could continue through to the mixed dentition stage and permanent dentition stage, cause facial asymmetry and invite temporomandibular joint dysfunction as well as modify the dental arch. Early treatment of subjects with deciduous dentition stage posterior cross bite is considered necessary, however the cause of the cross bite, craniofacial morphological characteristics and the position of individual teeth and tooth axes are still not fully understood and a consensus between experts on the ties between diagnosis and treatment is not necessarily seen. In this study we focused on the molar group tooth axes and analyzed models of 15 children with cross bite and models of children with healthy occlusion, compared the relationship between dental arch and tooth axis and examined palatal morphology and postero anterior cephalogram with the purpose of clarifying the aspect of posterior cross bite in the deciduous dentition stage. Results were as follows. In dental arch size measurement, there was a trend of the distance between each measurement being larger in the cross bite side of the cross bite group than the non-cross bite side. When compared with the control group, the distance in both the cross bite side and non-cross bite side of the cross bite group was significantly smaller. In maxillary and mandibular deciduous molar tooth axis measurement, no significant differences were observed in the maxillary and mandibular first and second deciduous molar tooth axis measurements between the cross bite group and the control group. In measurement of the angle between the maxillary and mandibular molar tooth axes (lingual), no significant differences were observed in the angle between the maxillary and mandibular first deciduous molar tooth axes and the angle between the maxillary and mandibular second deciduous molar tooth axes between the cross bite group and the control group. In palatal surface distance measurement, although there was no significant difference between the cross bite side and non-cross bite side of the cross bite group, the measurement of the control group was significantly higher than both the cross bite side and non-cross bite side of the cross bite group. In analysis by postero anterior cephalogram, no significant difference in maxillary and mandibular right and left jaw width and posterior symmetry was seen between the cross bite side and non-cross bite side of the cross bite group. The midline of the tooth deviated by $2.40 \mathrm{~mm}$ and the midline of the maxillary and mandibular jaw deviated by $3.25^{\circ}$ on the cross bite side in the cross bite group.
\end{abstract}




\section{Introduction}

Pediatric dentistry covers oral management during a stage of remarkable growth changes spanning the edentulous stage, deciduous dentition stage, mixed dentition stage and finally the permanent dentition stage. Understanding and predicting an individual's growth changes is extremely important in their oral management. In recent years there has been a growing interest in malocclusion in clinical pediatric dentistry as well as the treatment and prevention of caries and periodontal disease. The prediction of occlusion constitutes the core of clinical pediatric dentistry and thus it is important to seek related evidence.

One occlusal abnormality often observed in the deciduous dentition stage is posterior cross bite. Posterior cross bite in the deciduous dentition stage is classified as functional or skeletal depending on its pathogenesis, and many cases are functional (1). Posterior cross bite in the deciduous dentition stage is unlikely to heal on its own even as the jaw grows. If left untreated, it could continue through to the mixed dentition stage and permanent dentition stage, cause facial asymmetry and invite temporomandibular joint dysfunction as well as modify the dental arch $(2-5)$.

Previously we have measured the jaw movement of subjects with posterior cross bite in the deciduous dentition stage in our department and reported that it differs from the movement in children with healthy dentition (6). In addition, we have also carried out ear analysis through tympanometry and reported that the ears of subjects with posterior cross bite in the deciduous dentition stage can also be affected (7). Based on these reports, early treatment of subjects with deciduous dentition stage posterior cross bite is considered necessary, however the cause of the cross bite, craniofacial morphological characteristics and the position of individual teeth and tooth axes, etc. that effect jaw movement are still not fully understood and a consensus among experts on the ties between diagnosis and treatment is not necessarily seen $(8,9)$. Measurement of the maxillary and mandibular molar group tooth axes of children with posterior cross bite has not been undertaken in the past and thus no reports have been made comparing this aspect in children with posterior cross bite and children with healthy occlusion.

In this study we focused on the molar group tooth axes and analyzed models of children with cross bite and models of children with healthy occlusion, compared the relationship between dental arch and tooth axis and examined palatal morphology and postero anterior cephalogram (PA) with the purpose of clarifying the aspect of posterior cross bite in the deciduous dentition stage.

\section{Materials and Methods}

\section{Materials}

Dental impression model of 15 children (two boys and 13 girls, average age: five years, two months) diagnosed with unilateral cross bite and showing no caries or significant dental attrition in the the first and second maxillary and mandibular left and right deciduous molars (eight teeth) were used as the cross bite group and the oral models of 12 children (two boys and 10 girls, average age: five years, six months) with healthy deciduous dentition stage occlusion were used as a control group.

\section{Measurement methods :}

1) Dental arch size measurement

We measured the size of the dental arch by measuring the distance between each measuring point five times with electronic calipers (manufactured by NSK) and taking the average measurement after excluding the minimum and maximum values. Each measurement site of dentition width and maxillary and mandibular height is displayed in Fig. 1 and Fig. 2. Measurements were taken at the following locations based on the Japanese Society of Pediatric Dentistry's dental arch measurement sites (10).

(1) The distance between the maxillary and mandibular canine cusp on both sides $(\mathrm{C}-\mathrm{C})$

(2) The distance between the maxillary first deciduous molar buccal cusp on both sides (D-D)

(3) The distance between the mandibular first deciduous molar buccal cusp on both sides $\left(D^{-}-\mathrm{D}\right)$ 


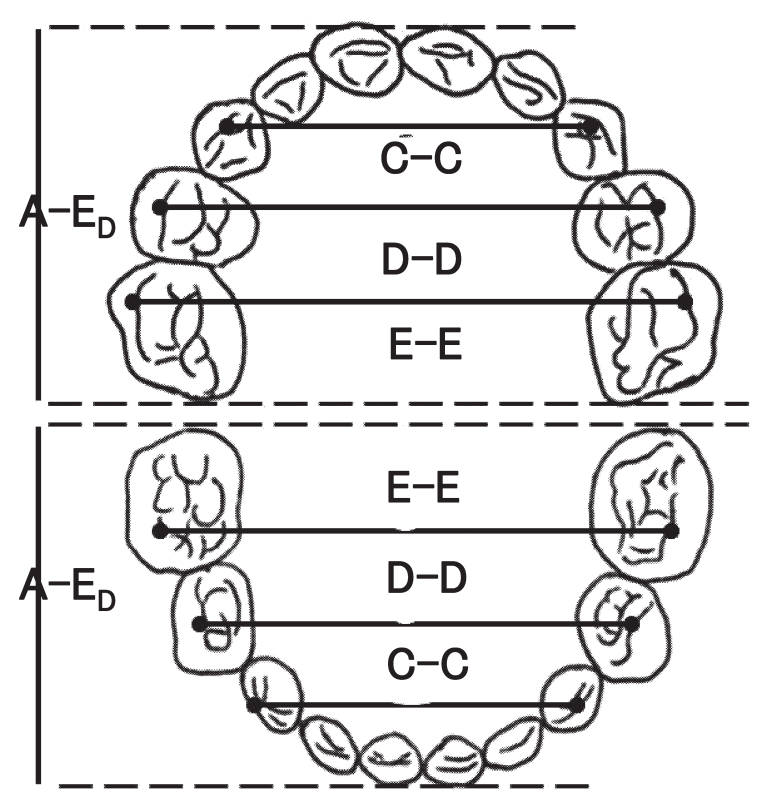

C-C : The distance between the maxillary and mandibular canine occlusal crown on both sides

D-D : The distance between the maxillary first deciduous molar buccal occlusal crown on both sides

The distance between the mandibular first deciduous molar buccal occlusal crown on both sides

E-E : The distance between the maxillary second deciduous molar buccal sulcus terminalis on both sides

The distance between the mandibular second deciduous molar mesial buccal sulcus terminalis on both sides

A-ED : The distance between the vertical line lowered from the centerof the line that extends through the facies labialis of the deciduous central incisor on both sides and the line connecting the most distal end of the second deciduous molar on both sides

Fig. 1. Dental arch size measurement

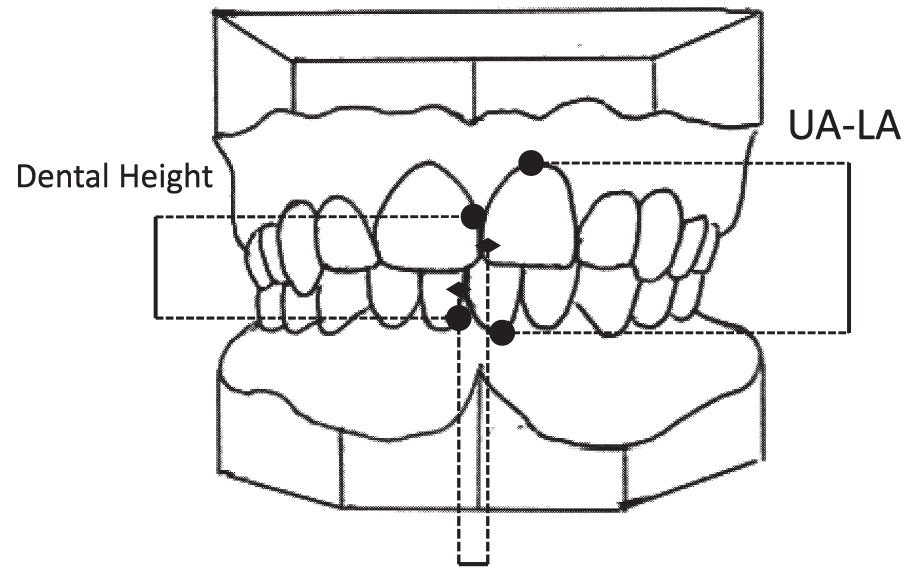

\section{Dental Height}

The distance between the maxillary and mandibular deciduous central incisor interdental papilla on both sides

\section{UA-LA}

The distance between the maxillary and mandibular deciduous central incisor facies labialis cervical center on both sides

The deviation of the maxillary and mandibular incisor midline

:The line through the point between mesial contacts of central primary incisors and interdental papilla

Fig. 2. Dental arch size measurement

(4) The distance between the maxillary second deciduous molar buccal groove terminalis on both sides $(\mathrm{E}-\mathrm{E})$

(5) The distance between the mandibular second deciduous molar mesial buccal groove terminalis on both sides $(\mathrm{E}-\mathrm{E})$

(6) The distance between the vertical line lowered from the center of the line that extends through the labialis of the deciduous central incisor on both sides and the line connecting the most distal end of the second deciduous molar on both sides (A-ED)

(7) The distance between the maxillary and mandibular deciduous central incisor interdental papilla on both sides (Dental Height)

(8) The distance between the maxillary and mandibular deciduous central incisor labialis cervical center on both sides (UA-LA)

(9) The deviation of the maxillary and mandibular 


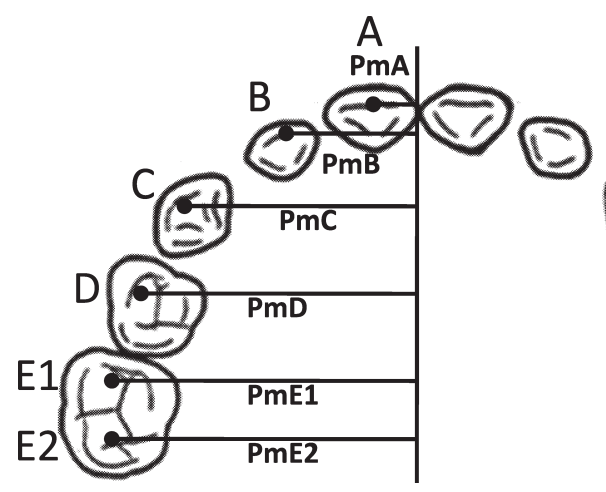

Palatal midline (Pm)

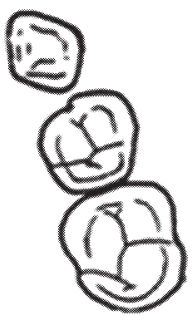

A : The midpoints of the mesiodistal crown width in the deciduous lateral and central incisors

B : The midpoints of the mesiodistal crown width in the deciduous lateral and central incisors

C : The peak of the deciduous canine

D : The buccal cusp of the first deciduous molars

E : The buccal cusp of the second deciduous molars

Fig. 3. Distance between the cusp and the palatal midline in the maxillary dental arch

\section{incisor midline}

The deviation of the maxillary and mandibular incisor midline was expressed using an absolute value.

2) Distance between the cusp and the palatal midline in the maxillary dental arch

The median palatal midline was taken as the palatal midline and the distance between the cusps of each tooth were measured and compared. The measurement sites are shown in Fig. 3. The midpoints of the mesiodistal crown width in the deciduous lateral and central incisors (A, B), the cuspid of the deciduous canine $(\mathrm{C})$ and the buccal cusp of the first and second deciduous molars (D, E1, E2) were made measurement points and a straight-line measurement from the palatal midline to each measurement point was carried out. We then compared the measurements of the cross bite side and non-cross bite side of the cross bite group with the control group.

3) Maxillary and mandibular deciduous molar tooth axis measurement

Maxillary and mandibular deciduous molar tooth axis measurement was carried out with a measuring device devised by Miyoshi et al (manufactured by Detect), the data was collected by Dipp Basic ${ }^{\circledR}$ and analyzed with Dipp Motion Pro ${ }^{\circledR}$ both of which are manufactured by the same company (Fig. 4). This measuring device takes images with three cameras and develops three-dimensional coordinates based on the coordinates displayed in each image. We performed tooth axis angle calculations by computer

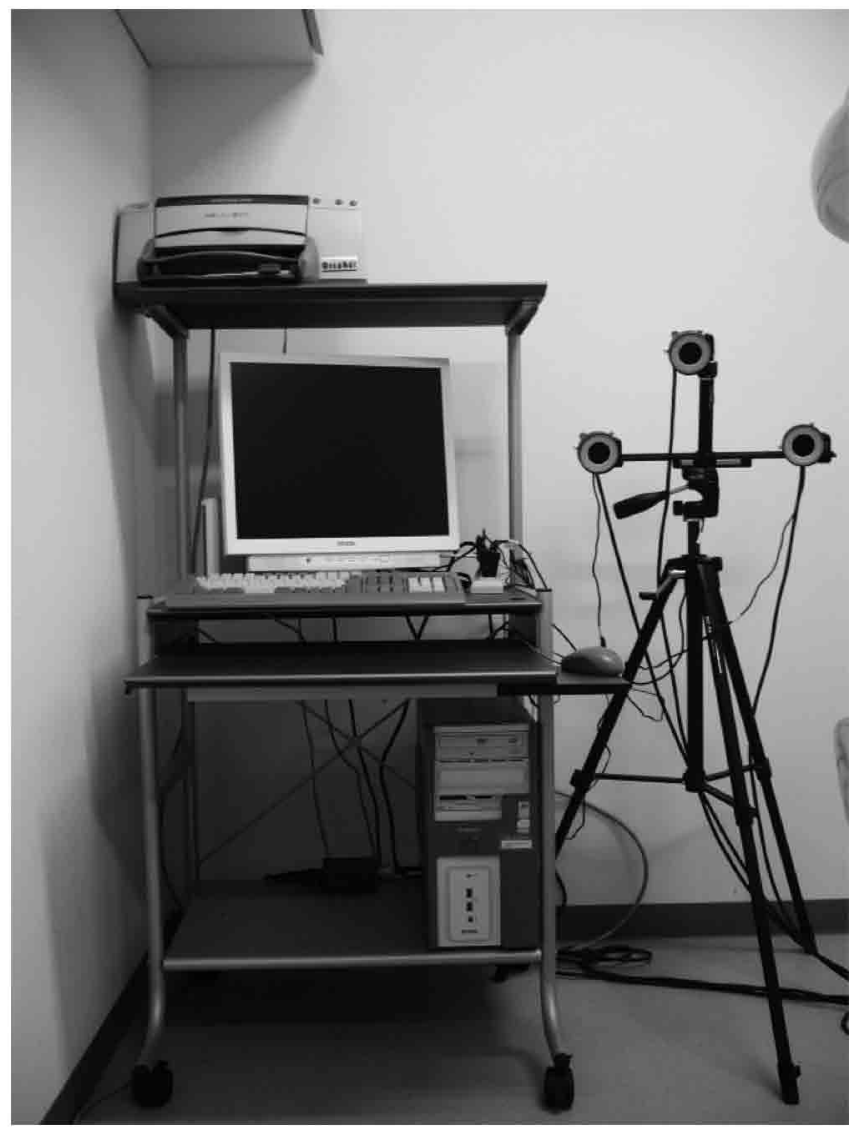

Fig. 4. Measuring device (manufactured by Detect)

based on the three-dimensional coordinate data.

The deciduous molar tooth axis was the angle formed by the orthogonal axes of the cervical plane and cusp plane of each molar. The maxillary and mandibular reference planes are shown below (Fig. $5)$. 

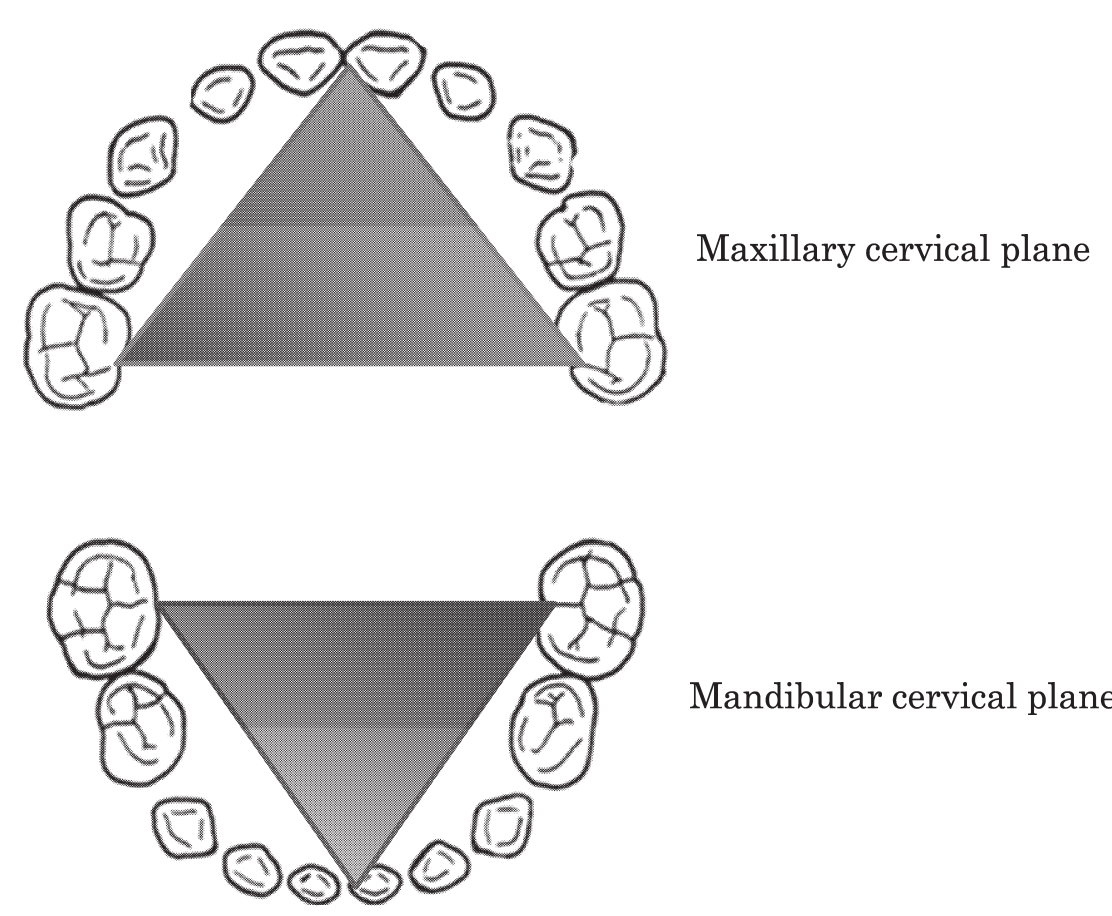

Mandibular cervical plane

Fig. 5. Maxillary and mandibular reference plane

a) Maxillary reference plane:

Maxillary cervical plane: A plane consisting of three points- the incisive papilla between the maxillary deciduous central incisors on both sides and the bottom of the cervical region of the deciduous second molars on both sides.

Maxillary deciduous first molar cusp plane: A plane consisting of the two points obtained by dividing the line passing through the maxillary deciduous first molar mesiodistal intersection and the buccal cusp region equally into three parts and the lingual cusp region.

Maxillary deciduous second molar cusp plane: A plane consisting of three points- the deciduous second molar mesiodistal and buccal cusps and mesiolingual cusp.

b) Mandibular reference plane:

Mandibular cervical plane: A plane consisting of three points-the papilla crown between the mandibular deciduous central incisors on both sides and the bottom of the cervical region of the deciduous second molars on both sides.

Mandibular deciduous first molar cusp plane: A plane consisting of three points- the deciduous first molar mesiodistal and buccal cusps and mesiolingual cusp.

Mandibular deciduous second molar cusp plane: A plane consisting of three points- the deciduous second molar mesiodistal and buccal cusps and distolingual cusp.

4) Deciduous molar tooth axis measurement

The angle between the deciduous molar tooth axis sought in 3) and cervical plane was sought by computer and the maxillary palatal side and mandibular lingual side was measured as the tooth axis angle to form the cervical plane. We then compared the measurements of the cross bite side and non-cross bite side of the posterior cross bite group with the control group (Fig. 6).

5) Measurement of the angle between the maxillary and mandibular molar tooth axes (lingual)

The lingual angle between the first and second maxillary and mandibular molar tooth axes was sought by computer and was compared between the cross bite side of the mouth and non-cross bite side of the mouth of the posterior cross bite group and the control group as the angle between the maxillary and mandibular molar tooth axes (Fig. 7). 


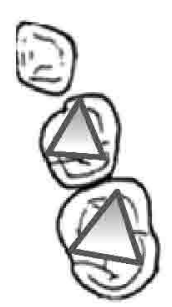

Maxillary deciduous first molar cusp plane Maxillary deciduous second molar cusp plane
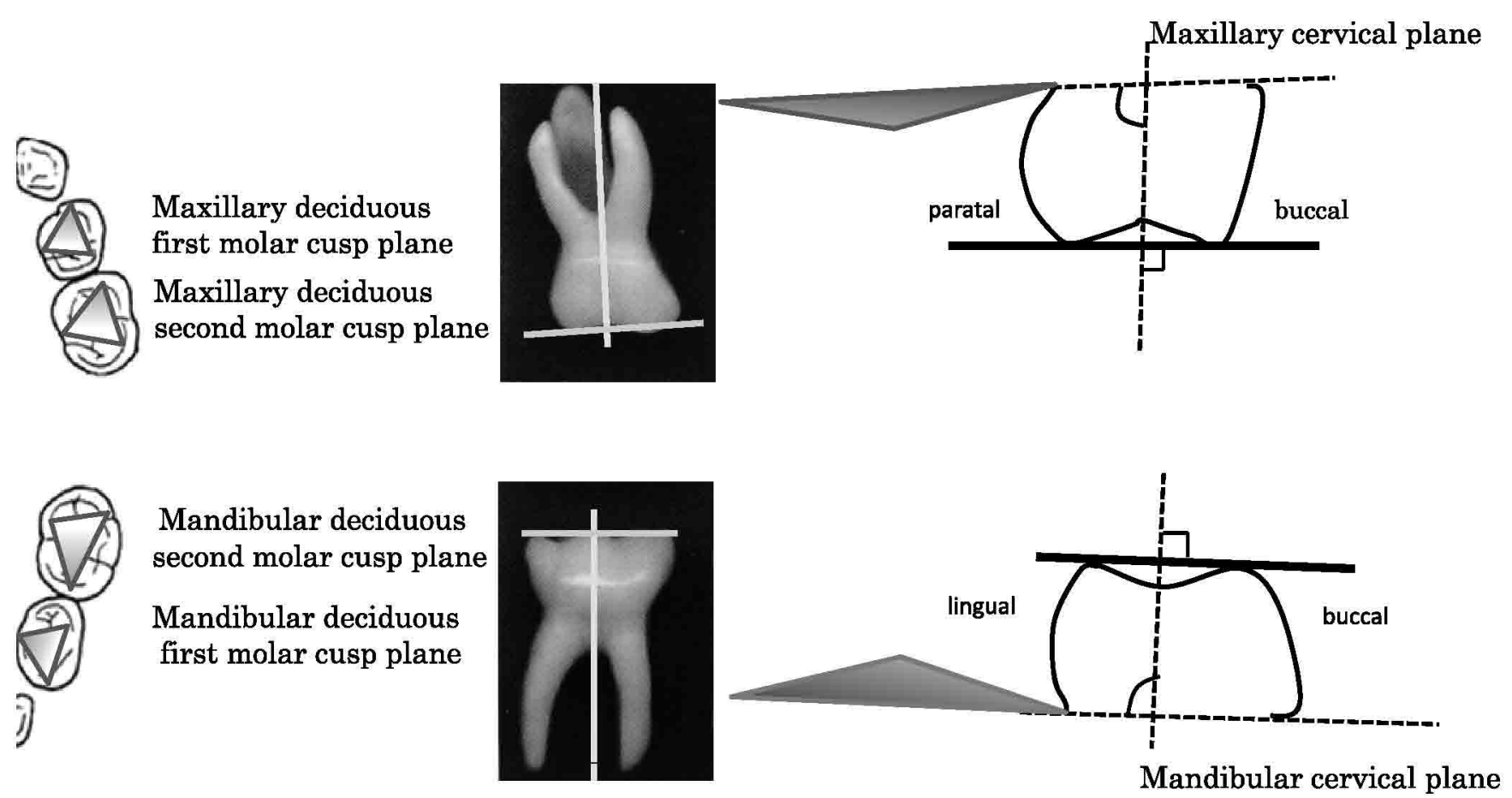

Fig. 6. Deciduous molar tooth axis measurement

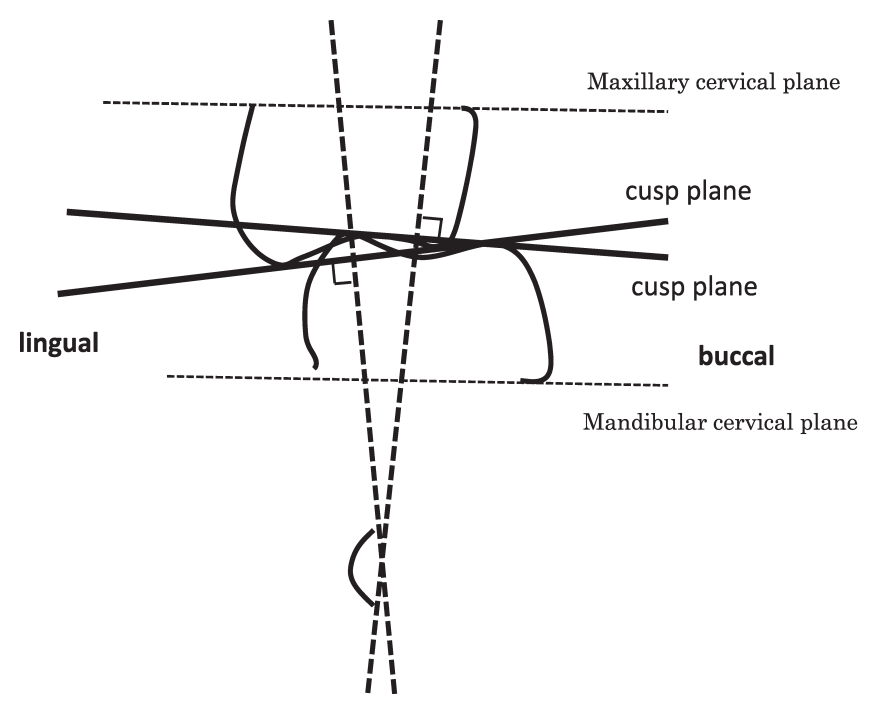

Fig. 7. Measurement of the angle between the maxillary and mandibular molar tooth axes (lingual)

\section{6) Analysis by postero anterior cephalogram}

Frontal head standard $\mathrm{x}^{-}$-ray photographs were used to determine whether the posterior cross bite was skeletal and the following measurements were carried out based on Ricketts analysis 22) and compared between the cross bite side and non-cross bite side of the posterior cross bite group (Fig. 8). (a) Reference line and measurement point setting CG-ANS : The line connecting the cranial median baseline, ethmoidal crest and anterior nasal spine

ZR, ZL : The intersection of the orbital rim and the interior frontozygomatic suture

ZA, AZ: Lateral midpoint of the zygomatic arch

JR, JL: The intersection of the maxillary tuberosity outline and zygomatic alveolar crest

AG, GA : The point outside the lower edge of the AG line

Front teeth plane: The plane connecting JR (JL) and GA (AG)

Front facial plane: The plane connecting ZR (ZL) and AG (GA)

(b) Measurement items

Tooth midline: The distance between the maxillary and mandibular deciduous central incisors used to determine functional deviation of the jaw, toothonly deviation from malocclusion, deviation from posterior cross bite or deviation from skeletal asymmetry.

Maxillary and mandibular left and right width : The distance between the maxillary and front face plane-used to determine whether the cross bite is 


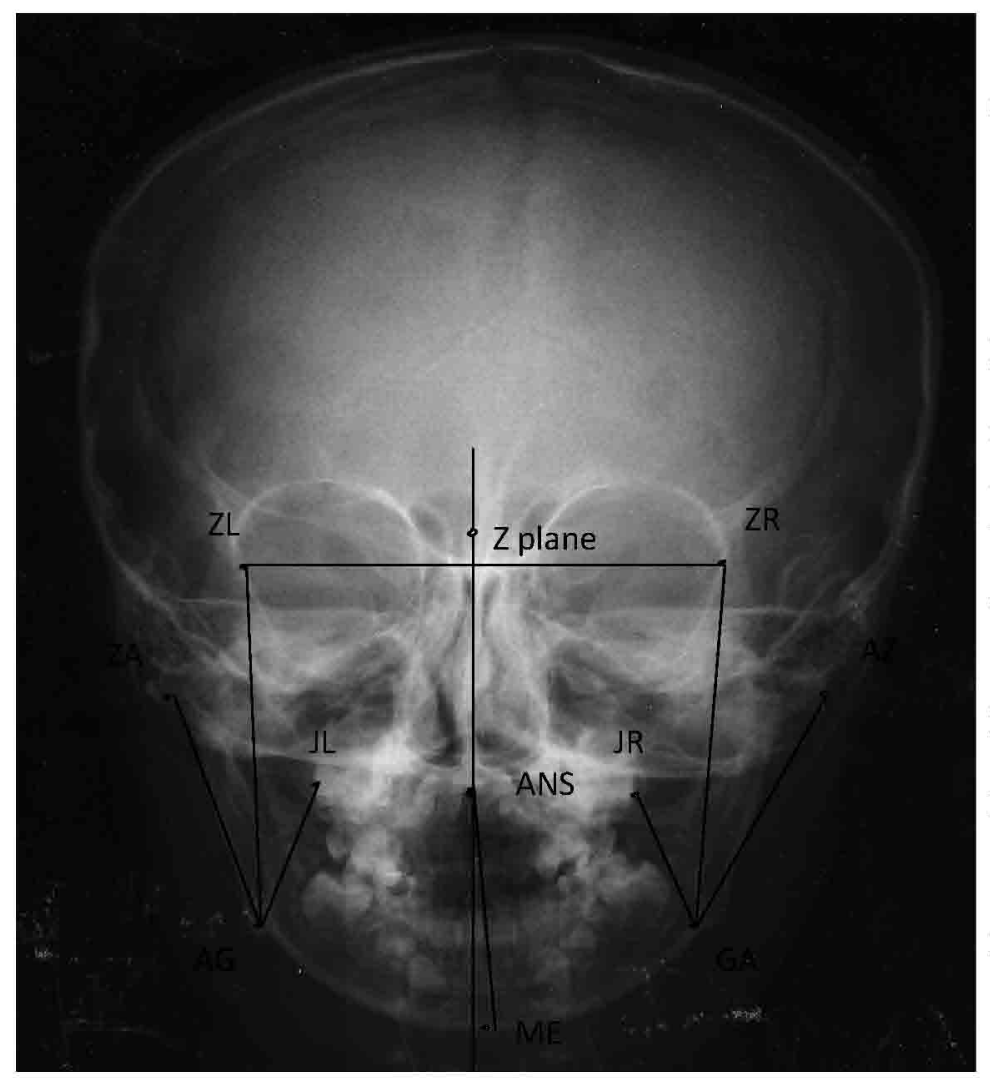

\section{Tooth midline}

The distance between the maxillary and mandibular deciduous central incisors - used to determine functional deviation of the jaw, tooth -only deviation from malocclusion, deviation from posterior cross bite or deviation from skeletal asymmetry.

Maxillary and mandibular left and right width

The distance between the maxillary and front face plane - used to determine whether the cross bite is skeletal. Large values indicate skeletal lingual cross bite and small values indicate skeletal buccal cross bite.

\section{Z plane}

The plane connecting ZR and ZL - a horizontal reference plane

\section{Maxillary and mandibular midline}

The angle between the plane where ANS-ME is perpendicular to the $\mathrm{Z}$ plane - used to determine differences in tooth size and whether there is functional median deviation.

\section{Posterior symmetry}

The ZR (ZL)-GA (AG)-AZ (ZA) angle on the left and right side - a large difference expresses skeletal asymmetry

Fig. 8. Analysis by frontal head standard $\mathrm{x}$-ray photographs

skeletal. Large values indicate skeletal lingual cross bite and small values indicate skeletal buccal cross bite.

$\mathrm{Z}$ plane: The plane connecting $\mathrm{ZR}$ and $\mathrm{ZL}-\mathrm{a}$ horizontal reference plane

Maxillary and mandibular midline: The angle between the planes where ANS-ME is perpendicular to the $Z$ plane - used to determine differences in tooth size and whether there is functional median deviation.

Posterior symmetry: The ZR (ZL)-GA (AG)-AZ (ZA) angle on the left and right side - a large difference expresses skeletal asymmetry.

7) Palatal surface distance measurement

Measurement was carried out with contact-type three-dimensional shape measuring equipment (3DPICZA, Inc. Roland, Hamamatsu) (Fig. 9) and the three-dimensional shape data were analyzed with three-dimensional image analysis software $\left(3 \mathrm{D}^{-}\right.$ RUGLE, Medic Engineering, Kyoto). Image J was used for measuring palatal surface distance. The

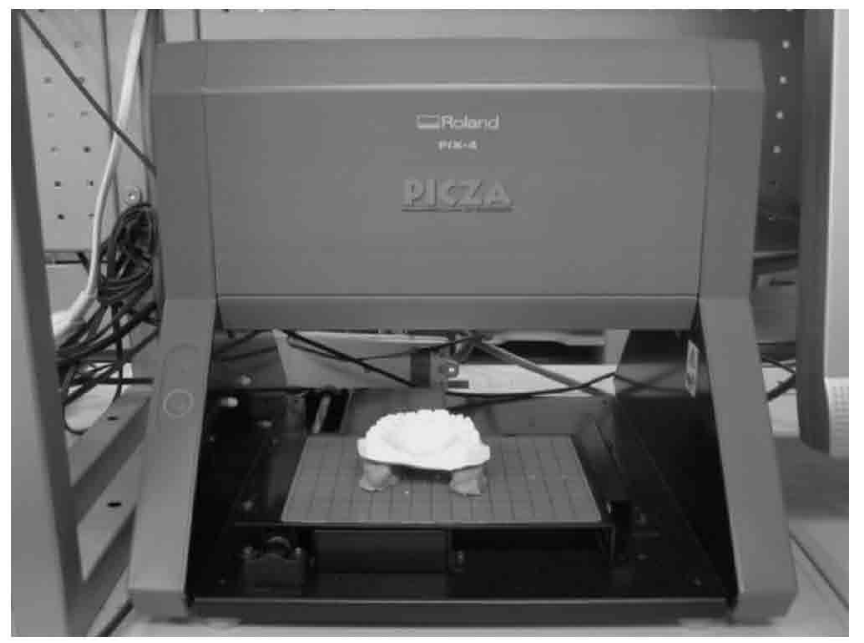

Fig. 9. Contact-type three-dimensional shape measuring equipment (3D-PICZA, Inc. Roland, Hamamatsu)

distance between the deepest cervical point of the second deciduous molar and the palatal midline was compared between the cross bite side and non-cross bite side of the cross bite group and the control group (Fig. 10). 


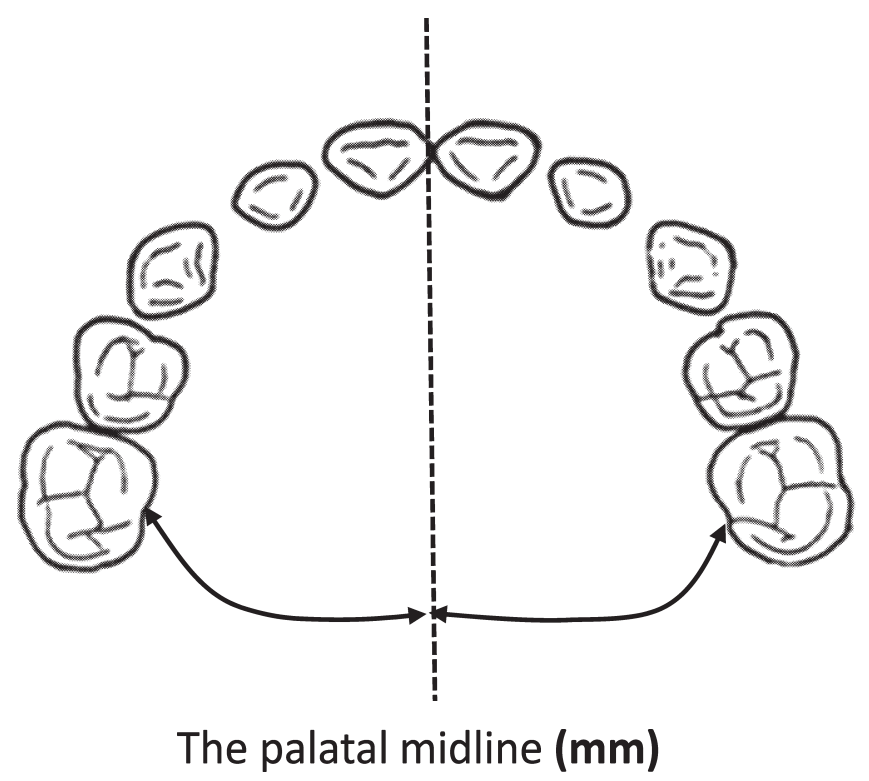

Fig. 10. Palatal surface distance measurement

\section{8) Statistical analysis}

The average value and standard deviation for each measurement item was calculated, the Mann-Whitney test used for statistical processing and the Bonferroni correction method used in multiple comparisons.

\section{Results}

1) Dental arch size measurement

The dental arch size measurement results are displayed in Table 1. The distance between the maxillary and mandibular canine cusp on both sides, the distance between the maxillary first molar buccal cusp on both sides and the distance between the maxillary second molar buccal groove terminalis on both sides were significantly narrower in the cross bite group than in the control group. A trend was observed of a larger mandibular dental arch in the cross bite group and a significant difference was seen between the groups in the distance between the mandibular second molar mesial buccal sulcus terminalis on both sides. The distance between the maxillary and mandibular deciduous central incisor inter-dental papilla on both sides (dental height) was significantly higher in the cross bite group. The distance between the maxillary and mandibular deciduous central incisor labialis cervical center on both sides (UA-LA) was significantly higher in the cross bite group on both the cross bite side and non -cross bite side than the control group. Finally, there was significantly greater deviation of the maxillary and mandibular incisor midline in the cross bite group (Table 2).

2) Distance between the cusp and the palate midline in the maxillary dental arch

The distance between the cusp and the palate midline in the maxillary dental arch is displayed in Table 3. There was no significant difference of the distance between each measurement in the cross bite side of the cross bite group and the non-cross bite side. When compared with the control group, the

Table 1. Dental arch size measurement

\begin{tabular}{|c|c|c|c|c|}
\hline \multirow{2}{*}{\multicolumn{3}{|c|}{ Measurement part }} & Normal occlusion & Cross bite \\
\hline & & & mean \pm S.D. & mean \pm S.D. \\
\hline \multirow{6}{*}{$\begin{array}{l}\text { Dental arch } \\
\text { weigh }\end{array}$} & \multirow{3}{*}{ Maxillary } & $\mathrm{C}-\mathrm{C}$ & $30.17 \pm 1.96 \stackrel{*}{*}$ & $-28.79 \pm 1.84$ \\
\hline & & $\mathrm{D}-\mathrm{D}$ & $38.50 \pm 2.44 \stackrel{*}{*}$ & $-36.19 \pm 2.22$ \\
\hline & & $\mathrm{E}-\mathrm{E}$ & $44.96 \pm 1.84$ & $-42.93 \pm 2.87$ \\
\hline & \multirow{3}{*}{ Mandible } & $\mathrm{C}-\mathrm{C}$ & $23.10 \pm 1.22$ & $24.17 \pm 2.06$ \\
\hline & & $\mathrm{D}-\mathrm{D}$ & $32.06 \pm 3.65$ & $33.23 \pm 2.35$ \\
\hline & & $\mathrm{E}-\mathrm{E}$ & $38.24 \pm 1.64$ * & $-39.89 \pm 2.32$ \\
\hline \multirow{2}{*}{$\begin{array}{l}\text { Dental arch } \\
\text { length }\end{array}$} & Maxillary & $A-E_{D}$ & $28.48 \pm 1.69$ & $28.81 \pm 1.83$ \\
\hline & Mandible & $A-E_{D}$ & $25.50 \pm 1.91$ & $24.86 \pm 1.37$ \\
\hline
\end{tabular}


Table 2. Dental arch size measurement

\begin{tabular}{|c|c|c|}
\hline \multirow{2}{*}{ Measurement part } & Normal occlusion & Cross bite \\
\cline { 2 - 3 } & mean \pm S.D. & mean \pm S.D. \\
\hline Dental Height & $4.52 \pm 1.25 * *$ & $6.40 \pm 1.02$ \\
\hline
\end{tabular}

\begin{tabular}{|c|c|c|c|}
\hline \multirow{3}{*}{$\begin{array}{c}\text { Measurement } \\
\text { part }\end{array}$} & \multirow{2}{*}{$\begin{array}{l}\text { Normal } \\
\text { occlusion }\end{array}$} & \multicolumn{2}{|c|}{ Cross bite } \\
\hline & & Cross bite side & Non-cross bite side \\
\hline & mean \pm S.D. & mean \pm S.D. & mean \pm S.D. \\
\hline UA-LA & $8.12 \pm 1.39$ & $* \quad 9.27 \pm 1.17$ & $9.68 \pm 1.22$ \\
\hline
\end{tabular}

\begin{tabular}{|c|c|c|}
\hline \multirow{2}{*}{ Measurement part } & Normal occlusion & Cross bite \\
\cline { 2 - 3 } & mean \pm S.D. & mean \pm S.D. \\
\hline $\begin{array}{l}\text { The deviation of the maxillary } \\
\text { and mandibular incisor midline }\end{array}$ & $0.57 \pm 0.49$ * $* *$ & $2.69 \pm 1.18$ \\
\hline
\end{tabular}

Table 3. Distance between the cusp and the palate midline in the maxillary dental arch

\begin{tabular}{|c|c|c|r|r|r|r|}
\hline & $\begin{array}{c}\text { PmA } \\
\text { mean } \pm \text { S.D. }\end{array}$ & $\begin{array}{c}\text { PmB } \\
\text { mean } \pm \text { S.D. }\end{array}$ & $\begin{array}{c}\text { PmC } \\
\text { mean } \pm \text { S.D. }\end{array}$ & $\begin{array}{c}\text { PmD } \\
\text { mean } \pm \text { S.D. }\end{array}$ & $\begin{array}{c}\text { PmE1 } \\
\text { mean } \pm \text { S.D. }\end{array}$ & $\begin{array}{c}\text { PmE2 } \\
\text { mean } \pm \text { S.D. }\end{array}$ \\
\hline $\begin{array}{c}\text { Normal } \\
\text { occlusion } \\
\mathrm{n}=12\end{array}$ & $3.52 \pm 0.28$ & $9.82 \pm 0.67$ & & & \\
\end{tabular}

$$
*: \mathrm{p}<0.05 \quad * *: \mathrm{p}<0.01
$$

distance in both the cross bite side and non-cross bite side of the cross bite group was significantly smaller.

3) Maxillary and mandibular deciduous molar tooth axis measurement (the palatal and lingual angles of the molar tooth axis and cervical plane)

The maxillary and mandibular deciduous molar tooth axis measurements are displayed in Table 4. No significant differences were observed in the maxillary and mandibular first and second deciduous molar tooth axis measurements between the cross bite group and the control group.

4) Measurement of the angle between the maxillary and mandibular deciduous molar tooth axes (lingual)

The measurements of the angle between the maxillary and mandibular molar deciduous tooth axes are displayed in Table 4. No significant differences were observed in the angle between the maxillary and mandibular first deciduous molar tooth axes and the angle between the maxillary and mandibular second 
Table 4. Maxillary and mandibular deciduous molar tooth axis measurement (the palatal and lingual angles of the molar tooth axis and cervical plane)

(angle)

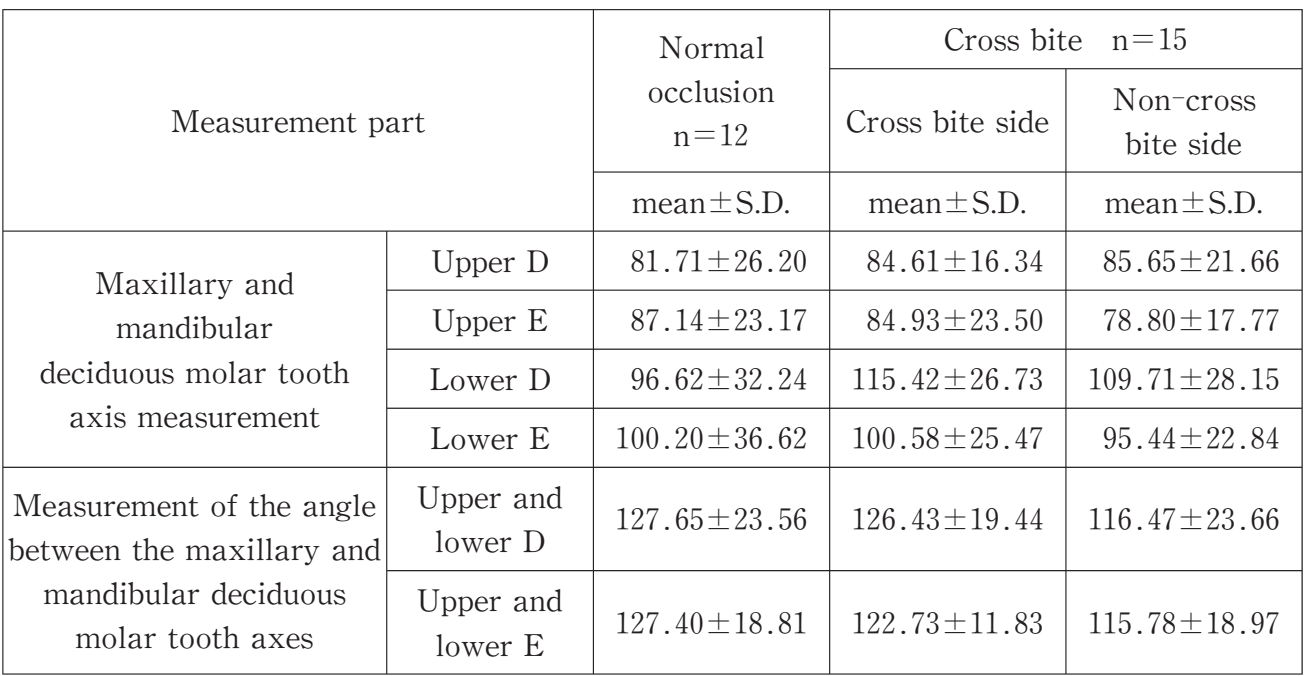

Table 5. Palatal surface distance measurement

\begin{tabular}{|c|c|c|c|}
\hline \multirow{2}{*}{$\begin{array}{c}\text { Measurement } \\
\text { part }\end{array}$} & $\begin{array}{c}\text { Normal } \\
\text { occlusion }\end{array}$ & \multicolumn{2}{|c|}{ Cross bite } \\
\cline { 2 - 4 } & mean \pm S.D. & mean \pm S.D. & mean \pm S.D. \\
\cline { 2 - 4 } $\begin{array}{c}\text { Palatal surface } \\
\text { distance }\end{array}$ & $20.6 \pm 2.02$ & $17.89 \pm 1.81$ & $17.69 \pm 1.33$ \\
\hline
\end{tabular}

$* *: \mathrm{p}<0.01$

Table 6. Postero-anterior cephalogram

\begin{tabular}{|c|c|c|}
\hline \multirow{2}{*}{ Measurement part } & $\begin{array}{c}\text { Maxillary and mandibular } \\
\text { left and right width }(\mathrm{mm})\end{array}$ & $\begin{array}{c}\text { Posterior symmetry } \\
\text { (angle) }\end{array}$ \\
\cline { 2 - 3 } & mean \pm S.D. & mean \pm S.D. \\
\hline Cross bite side & $11.50 \pm 1.98$ & $19.54 \pm 4.75$ \\
\hline Non-cross bite side & $10.92 \pm 2.54$ & $17.75 \pm 2.73$ \\
\hline
\end{tabular}

\begin{tabular}{|c|c|c|}
\hline \multirow{2}{*}{ Measurement part } & Tooth midline $(\mathrm{mm})$ & $\begin{array}{c}\text { Maxillary and mandibular } \\
\text { midline (angle) }\end{array}$ \\
\cline { 2 - 3 } & mean \pm S.D. & mean \pm S.D. \\
\hline cross bite group & $2.40 \pm 1.02$ & $3.25 \pm 1.19$ \\
\hline
\end{tabular}

deciduous molar tooth axes between the cross bite group and the control group.

5) Palatal surface distance measurement

A comparison of the palatal surface distance from the deepest cervical point of the second deciduous molar to the palatal midline between the cross bite side and non-cross bite side of the cross bite group and the control group is displayed in Table 5. 
Although there was no significant difference between the cross bite side and non-cross bite side of the cross bite group, the measurement of the control group was significantly higher than both the cross bite side and non-cross bite side of the cross bite group.

6) Analysis by postero anterior cephalogram

The results of analysis by frontal head standard $\mathrm{x}$ -ray photographs are shown in Table 6. No significant difference in maxillary and mandibular right and left jaw width and posterior symmetry was seen between the cross bite side and non-cross bite side of the cross bite group. The midline of the tooth deviated by $2.40 \mathrm{~mm}$ and the midline of the maxillary and mandibular jaw deviated by $3.25^{\circ}$ on the cross bite side in the cross bite group.

\section{Discussion}

The number of patients with the main complaint of malocclusion during the deciduous dentition stage has been increasing in clinical pediatric dentistry in recent years. If the treatment of deciduous dentition stage malocclusion is performed during the deciduous dentition stage, the exchange period is completed. However, there are also cases where treatment is carried out once the permanent dentition stage is reached and the period of treatment is currently under debate $(5,15-17)$.

Deciduous dentition stage posterior cross bite is an uncommon form of deciduous dentition stage malocclusion (18). However, because it more difficult for parents to detect compared with anterior cross bite and even difficult to detect in school physical examinations, it is often left untreated until the mixed dentition and permanent dentition stages. It is also believed that posterior cross bite is less likely to heal on its own than anterior cross bite. Shimada et al. (19) examined changes in the occlusal state of children between the age of 3-5 years and reported that maxillary protraction, anterior cross bite, overbite, open bite and crowding decreases by age five compared with age three while the incidence of incisal edge to edge occlusion and cross bite increases.

Cuspal interference of deciduous canines due to constriction of the maxillary dental arch is reported as a cause of deciduous dentition stage posterior cross bite. Ono reports that unilateral cross bite which is most frequent in the mixed dentition stage is often already seen in the deciduous dentition stage and that cuspal interference of deciduous canines is often observed for some reason (20). Graber reports the occurrence of deciduous canine cuspal interference along with narrowing of the maxillary dental arch and the development of gaps in the maxillary and mandibular front teeth in cases resulting from significant finger sucking (21) and Ricketts has identified that constriction of the maxillary dental arch is caused as a result of the anterior location of the tongue in cases where there is a habit of tongue protrusion, resulting in further narrowing of the palate and cross bite in the molar region (22). In addition, crown size $(23,24)$ and jaw morphology (25) have also been cited as factors influencing dental arch morphology.

Deciduous dentition stage posterior cross bite is classified into functional and skeletal cases according to pathogenesis and many posterior cross bite cases in deciduous dentition stage are functional cases. Moyers reported that observing the path of bite closure to differentiate between cases of unilateral functional and skeletal cross bite (26). Essentially, in unilateral cross bite the mandible is shifted to one side in a position of intercuspation and there is no deviation of the mandible from the position of closure to the position of maximum opening when there is deciduous canine cuspal interference. In skeletal cross bite, a deviation of the midline to one side occurs before contact of the teeth and problems with the underlying bone are sometimes reported in cases where there is a large shift to the side at the point of maximum opening.

If deciduous dentition stage posterior cross bite is left untreated, the cross bite could persist into the mixed dentition and permanent dentition stage casing facial asymmetry as well as deformation of the dental arch and inviting the risk of temporomandibular joint dysfunction (2-5).

1) Subjects

The 15 children that were the subjects of this study 
had been diagnosed with $f$ unilateral cross bite and showed no caries or significant dental attrition in the first and second maxillary and mandibular left and right deciduous molars (eight teeth). In terms of jaw movement, they didn't have any obvious mandible deviation from the position of closure to the position of maximum opening. In addition, deciduous canine edge-to-edge occlusion and cross bite was observed on the cross bite side of all these subjects. The control group consisted of 12 children with healthy deciduous dentition stage occlusion whose ages corresponded with the cross bite group.

\section{2) Dental arch morphology}

There are many reports that measure the distance between cuspids in the canine region, and many reports that measure the distance between cusps or between pits and fissures in the molar region (10-13) for the purpose of dental arch width measurement in this study. In the dental arch measurements, the width of the maxillary dental arch was narrower in the cross bite group and significant differences were also seen between the groups in the distance between the maxillary canine cusp on both sides, the distance between the maxillary first molar buccal cusp on both sides and the distance between the maxillary second molar buccal groove terminalis on both sides.

In the maxillary and mandibular dental arch, a significantly smaller distance between the cusp and palatal midline was observed in both the cross bite side and non-cross bite side of children with cross bite in comparison to the healthy occlusion children and there was a tendency for children with cross bite to have a constricted maxillary dental arch with greater constriction on the cross bite side.

Children with cross bite tended to have a larger mandibular dental arch in comparison to the children with healthy occlusion and a significant difference was seen between the two groups in the distance between the mandibular second molar mesial buccal groove terminalis on both sides. A shift of the mandibular midline to the cross bite side in children with cross bite was observed and a significant difference between the groups was also seen in maxillary and mandibular incisor midline deviation.
In general, deciduous dentition stage cross bite often causes constriction of the maxillary dental arch and deciduous canine cuspal interference. In addition, there are also cases resulting in caries, unilateral chewing and habitual mandibular lateral deviation due to sleeping posture. Deciduous dentition stage posterior cross bite is suggested to transition from functional to skeletal and cause facial asymmetry and temporomandibular joint dysfunction.

\section{3) Molar tooth axis}

No significant difference was seen between the cross bite group and normal occlusion group in maxillary and mandibular first and second deciduous molar measurement in molar tooth axis angle mean and standard deviation and significance test results. In the angle between the maxillary and mandibular deciduous molar tooth axes, the angles between the maxillary and mandibular first deciduous molar and the maxillary and mandibular second deciduous molar axes tended to be larger in the cross bite group; however this didn't amount to a significant difference.

Although maxillary dental arch constriction was observed in the cross bite group, no significant difference was seen in tooth axis. Because of this, it was suggested that the occlusal state of children with cross bite is primarily a deviation to the mandibular cross bite side as a result of early maxillary and mandibular deciduous canine contact. In addition, it was suggested that because there was no significant difference in maxillary and mandibular deciduous molar tooth axis angles between the cross bite side and the non ${ }^{-}$cross bite side in children with cross bite as well as between the children with cross bite and children with healthy occlusion, posterior cross bite is not involved with the deciduous molar tooth axis.

\section{4) Postero anterior cephalogram}

Postero anterior cephalogram were used in this study to determine mandibular deviation and whether the cross bite was skeletal in children with cross bite. We took measurements based on Ricketts analysis (22) and compared the cross bite and noncross bite groups. When we compared the maxillary 
and mandibular left and right width and posterior symmetry between the cross bite side and non-cross bite side of the cross bite models, no craniofacial skeletal abnormalities were observed from the postero anterior cephalogram. However, a deviation of tooth and maxillary and mandibular midline to the mandibular cross bite side was observed. Because deviation to the cross bite side was observed in deciduous dentition stage posterior cross bite, it is thought that cross bite may secondarily induce future asymmetric mandibular growth and cause skeletal lateral deviation of the mandible.

5) Palatal surface distance measurement

A significant difference in palatal vault distance measurement was observed between the cross bite group and the healthy occlusion group. Constriction was also observed in the molar region of the maxillary dental arch in the cross bite group. No specific differences were observed between the cross bite side and non-cross bite side of the cross bite group. Accordingly, it was thought that constriction doesn't just occur on either the cross bite side or the noncross bite side, but rather on both sides. In addition, there were not considered to be any skeletal abnormalities in the maxillary dental arch constriction occurring with cross bite.

From the results of this study it was thought that constriction of the maxillary dental arch observed from dental arch morphology and accompanying constriction of the deciduous molar region on the cross bite side, maxillary and mandibular dental arch size disharmony and mandibular deviation is highly likely to occur with cross bite. Cross bite is not represented by one specific factor; rather it is thought to be represented by the effect of a combination of individual factors. It was also thought that cross bite doesn't affect the degree of maxillary and mandibular deciduous molar tooth axis Inclination.

Although it is debated whether treatment for malocclusion, etc. should be carried out in the deciduous dentition stage, from the past reports of this department $(6,7)$ and from the standpoint of growth and development, treatment commenced during the deciduous dentition stage is considered easy and good therapeutic results are obtained. Kutin observed 35 cases of deciduous dentition stage posterior cross bite without orthodontic treatment and reported that the first deciduous molar did not erupt in the normal position in 32 of these subjects (27). As a result of this, it is thought that mixed dentition stage posterior cross bite is cross bite that already existed in the deciduous dentition stage and continued on to effect the state of dentition in the mixed dentition stage. Tsujino observed the dental arch width of 28 children from the age of three to the age of 20 using progressive maxillomandibular models obtained every two months (28) and reported an observation that maxillary inter-canine width increased between the ages of six and eight until the first and second maxillary deciduous molars fell out.

Malocclusion accompanying maxillary constriction can pose functional and aesthetic problems and there are two methods of effective treatment: rapid maxillary expansion and slow, gentle expansion. In rapid maxillary expansion, a fixed expansion device incorporating expanding screws is used and intermittent force is applied. Through the lateral expansion of the maxillary molar region over a short time, median palatal suture dehiscence and expansion of the maxilla itself primarily through the ossification of this gap can be expected (29-33). The method of slow, gentle expansion applies gentle force to the median palatal suture with the purpose of achieving expansion from a more physiological response than the rapid expansion method (34-36).

Maxillary dental arch constriction was the main feature of deciduous dentition stage posterior cross bite from this study and it was thought that accompanying maxillary and mandibular dental arch size disharmony and mandibular deviation is highly likely to occur with cross bite. In addition, although midline deviation was observed in the maxillary dentition, the deciduous molar tooth axis didn't affect the occlusal condition of children with cross bite and no significant difference in the distance from the midline was observed between the cross bite side and the non-cross bite side despite constriction of the maxillary dental arch in the residual ridge being 
observed. Because of this, the important point in treating cross bite was considered to be the expansion of the maxillary dental arch with treatment focused on the median palatal suture dehiscence rather than the application of orthodontic force to the teeth. The elimination of premature contact that may lead to lateral mandibular deviation was also considered necessary at this stage.

Improving maxillary and mandibular dental arch size disharmony during the deciduous dentition period leads to a future increase in the width of maxillary dentition. Improving the occlusal condition on both the cross bite side and the non-cross bite side and guiding the mandible into its appropriate position was thought to lead to the prevention of future jaw deviation and temporomandibular joint abnormalities.

\section{Acknowledgments}

We are grateful to Prof. T Maeda of the Department of Pediatric Dentistry in Nihon University School of Dentistry at Matsudo for his valuable advice.

\section{References}

1. Yamada H, Akasaka M: Basic and clinic in minor tooth movement, Dental Diamond, Tokyo, 1991, p.194 -196. (Japanese)

2. Kobayashi M, Saito I, Ishii K, Takehara M, Morita S, Hanada K: Lomg-term Observation of Mandibular Protrusion Cases Worsen in Pubertal Growth Spurt. J Jpn Orthod Soc, 55 : 234-245, 1996.

3. Kurihara K, Fujita Y, Soma K: An Investigation into the Relationship between Lateral Displacement of the Mandible and the Symptomatic Side. J Jpn Soc TMJ, 8: 36-46, 1996.

4. Usami R, Tanne K: Malocclusion Class III in clinic. Ishiyaku Publishers Inc, Tokyo, 1997, p.260-272. (Japanese)

5. Boer DE, Steenks MH : Functional Unilateral Posterior Crossbite. Orthodontic and Functional Aspects. J Oral Rehabilitation, 24: 614-623, 1997.

6. Miyoshi K : Cross bite and the jaw movement. Jpn J Clin Dent Child, 12-7 : 59-66, 2007. (Japanese)

7. Atsuko N: Evaluation of Middle Ear Function in Primary Dentition Children with Posterior Cross bite. Ped Dent J, 19-1 : 58-67, 2009.

8. Inoue N : Radiographic Observation of Rapid Expan- sion of Human Maxilla. Bull Tokyo Med Dent Univ, $17: 249-261,1970$.

9. Walters, RD : Facial Changes in the Macaca Mulatta Monkey by Orthopedic Opening of the Midpalatal Suture. Angle Orthodont, 45 : 169-179, 1975.

10. Japanese Society of Pediatric Dentistry: Research Concerning the Size of the Primary Tooth Crown, Primary Dental Arch and the Condition of Primary Occlusions of the Japanese. Jpn J Ped Dent, 31: 375388, 1993.

11. Barrow, GV, White, JR: Developmental Changes of the Maxillary and Mandibular Dental Arches. Angle Orthod, 22 : 41-46, 1952.

12. Moorrees, CFA : The Dentition of the Growing Child, 1st ed, 87-110, Harvard Univ press, Cambridge, 1959.

13. Moorress, CFA, Chadha, JM : Available Space for the Incisors During Dental Development-A Growth Study Based on Physiologic Age. Angle Orthod, 35 : 12-22, 1965.

14. Sinclair, PM, Little, RM: Maturation of Untreated Normal Occlusions. Am J Orthod, 83 : 114-123, 1983.

15. Thilander BW, Wahlund S, Lennartsson B: The Effect of Early Interceptive Treatment in Children with Posterior Cross-Bite. Europe J Orth, 6: 25-34, 1984.

16. Peter W Ngan, Stephen HY Wei : Treatment of Posterior in the Primary and Early Mixed Dentitons. Quintessence international, 21: 451-459, 1990.

17. Kurol J, Lena Berglund: Longitudinal Study and Costbenefit Analysis of the Effect of Early Treatment of Posterior Cross-Bite in the Primary Dentition, Europe. J Orth, 14 : 173-179, 1992.

18. Tanakamaru H, Machida Y : Examination and diagnosis in primary dentition. Dental Diamond, Tokyo, 1991, p.158-169. (Japanese)

19. Shimada M, Tonogi N, Yonezu T, Saijyou T, Machida Y : A Study on Occlusal Changes in Children from 3 to 5 Years of Age. Shika Gakuho, 96 : 951-955, 1996.

20. Ohno Y, Kanemoto H : Cross bite in mixed dentition. Dental Journal, $11: 337-350,1980$. (Japanese)

21. Graber, TM : orthodontics-Principles and Practice, 1972, WB Saunders Co, Philadelphia, USA, p.847-853.

22. Ricketts RM : Ethetics, Environment, and the Law of Lip Relation Amer. J Orthod, 54 : 272-289, 1968.

23. Lundstrom, A: The Etiology of Crowding of the Teeth. Trans Eur Orthod Soc, 25 : 176-191, 1951.

24. Doris, JM, Bernard, DW, Kuftinec, MM : A Biometric Study of Tooth Size and Dental Crowding. Am J Orthod, 79: 326-336, 1981.

25. Otsubo J : A Study on the Tooth Material in Japanese Adults of Normal Occlusion, Its Relationship to Coronal and Basal Arches. J Jpn Orthod Soc, 24 : 1-7, 1957.

26. Moyers, P.E: Handbook of Orthodontics, 1988, Year Book Medical Publishers, Inc, Chicago, USA, p.149- 
151.

27. Kutin, G, Hawes RR: Posterior Cross-Bite in the Deciduous and Mixed Dentitions. Amer J Orthodont, 56: 491-504, 1969.

28. Tujino K, Machida Y : A Longitudinal Study on the Growth and Development of the Dental Arch Width from Childhood to Adolescence. Jpm J Ped Dent, 35 : 670-683, 1997.

29. Angell, EH : Treatment of Irregularity of the Permanent of Adult Teeth. Dent Cosmos, 1: 540-544, 1860.

30. Korkhaus G: Present Orthodontic Thought in Germany Jaw Widening with Active Appliances in Cases of Mouth Breathing. Am J Orthod, 46 : 187-206, 1960.

31. Haas, AJ : Palatal Expansion; Just the Beginning of Dento-facial Orthopedics. Am J Orthod, 57 : 219-255,
1970.

32. Wertz, RA: Skeletal and Dental Changes Accompanying Rapid Midpalatal Suture Opening. Am J Orthod, 58: 41-66, 1970.

33. Iizuka T, Iwasawa T, Sebata M, Motohashi Y : Orthodontics, Third edition, Ishiyaku Publishers. Inc Tokyo, 1991, 233-234.

34. Storey, E: Tissue Response to the Movement of Bones. Am J Orthod, 64 : 229-247, 1973.

35. Cotton, LA : Slow Maxillary Expansion: Skeletal Versus Dental Response to Low Magnitude Force in Macaca Mulatta. Am J Orthod, 73 : 1-23, 1978.

36. Hicks, EP : Slow Maxillary Expansion: A Clinical Study of the Skeletal Versus Dental Response to Low -Magnitude Force. Am J Orthod, 73 : 121-141, 1978. 